\title{
High Selectivity Boolean Olfaction Using Hollow-Core Wavelength- Scalable Bragg Fibers
}

\author{
Mecit Yaman, ${ }^{\dagger, \ddagger}$ Adem Yildirim, ${ }^{\dagger, \ddagger}$ Mehmet Kanik, ${ }^{\dagger, \ddagger}$ Tugrul C. Cinkara, ${ }^{\dagger, \ddagger}$ and Mehmet Bayindir $*, \dagger, \ddagger, \S$ \\ ${ }^{\dagger}$ UNAM-National Nanotechnology Research Center, ${ }^{\ddagger}$ Institute of Materials Science and Nanotechnology, and ${ }^{\S}$ Department of \\ Physics, Bilkent University, 06800 Ankara, Turkey
}

\section{Supporting Information}

\begin{abstract}
A new odorant detection scheme, based on infrared absorption of volatile organics inside an optofluidic channel array, is discussed in terms of its selectivity. The sensor unit of the array is a hollow core Bragg fiber that selectively (spectrally) guides an incident continuum radiation. The presence of infrared absorbing molecules in the channel results in the quenching of the otherwise transmitted signal.

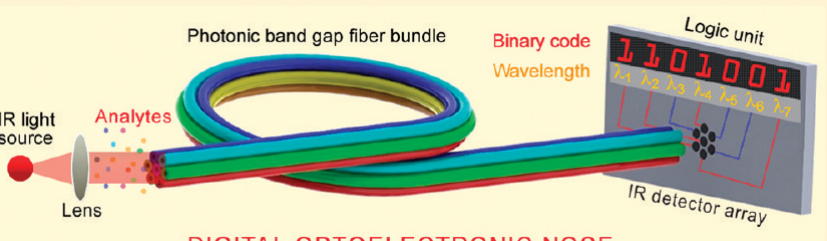

DIGITAL OPTOELECTRONIC NOSE Each fiber unit in the array is designed and fabricated so that it is sensitive to specific chemical bonds and the bond environment, but at the same time, each fiber is also broadly sensitive to a large number of chemicals due to their infrared absorbance spectra. The cumulative array response data, using an appropriate threshold, enable selective binary sampling of the infrared fingerprint of hundreds of molecules. The selectivity of the system is quantitatively investigated with computer simulations and found to be exponentially increasing with the number of fibers in the array. Relatively simple data analysis using binary logic combined with the high selectivity of the novel scheme paves the way for ubiquitous application of electronic noses in toxic gas detection, food quality control, environmental monitoring, and breath analysis for disease diagnostics.
\end{abstract}

A rtificial nose research involves mimicking vertebrate olfaction, where airborne analytes are sensed by an array of sensory receptors, followed by the processing of the cumulative response in the olfactory bulb in order to distinguish tens of thousands of odorants with exceptional sensitivity and selectivity. ${ }^{1,2}$ Numerous transducing mechanisms have been employed to make an electronic nose, including metal oxide semiconductors and FETs, ${ }^{3,4}$ chemiresistors, 3,6 and polymer coated micro- and nanocantilever arrays. ${ }^{7,8}$ Optical transducing schemes are also gaining ground, such as fluorescent dye coated fiber optic microarrays, ${ }^{9-11}$ organic dye arrays, ${ }^{12-14}$ and mesoporous Bragg layers. ${ }^{15}$

The recent advent of photonic technologies, such as the availability of high power tunable lasers, infrared sources and detectors, and photonic band-gap waveguide channels, and the convergence of microfluidics and photonics are driving new optical detection schemes in chemosensing and optofluidics. ${ }^{16}$ Molecular infrared absorption ${ }^{17}$ is one such technique that became ever more pertinent ${ }^{18}$ in sensing organic molecules based on their spectral absorption fingerprint. One immediate advantage of IR absorption spectroscopy over chemiresistance or adsorption based transducing is that the gas cell can be reset anytime by flushing and can be used with little or no aging effect.

Conventional Fourier transform infrared (FTIR) spectroscopy has sensitivities in the sub parts per million (ppm) analyte concentration. ${ }^{17}$ This limit can be improved by, for example, plasmonic enhancement by antenna arrays to detect, in some cases, single molecules. ${ }^{19}$ Optofluidic channels bring together molecular selectivity and sensitivity of infrared absorption spectroscopy, with enhanced electromagnetic interactions possible in planar or fiber waveguides. Using optofluidic channels, sensitivity can be dramatically increased, minimal volume analyte sampling can be exploited, and response time can be brought down to real time. Already, hollow core metallic and dielectric waveguides and fibers were used as a capillary gas flow channel that serves both as the gas cell and as a waveguide for an incident beam. ${ }^{20}$ Although very high sensitivity can be achieved using a laser beam, the selectivity of these schemes is limited to single species, constrained by the line spectra of laser sources and the narrow tunability of the sensors.

Recently, we demonstrated that high selectivity chemical sensing in hollow core photonic band-gap (PBG) fibers can be performed with a continuum blackbody source rather than a narrow band high power laser. ${ }^{21}$ Such design enables detection of any infrared absorbing chemical based on the detection of its infrared absorption pattern by spanning the whole spectrum with the sensing fiber array. The specialty PBG fibers are produced, by controlling fiber drawing parameters, to guide a specified spectral band from the whole infrared spectrum relevant to organic molecules. ${ }^{21}$ In this scheme, therefore, fibers are used not only as a waveguide or absorption cell but also as a filter for the incoming infrared radiation by virtue of their wavelength-scalable photonic band structure. The PBG fiber transmits a specific infrared band determined by the Bragg layer

Received: June 10, 2011

Accepted: November 28, 2011

Published: November 28, 2011 
Scheme 1. Schematic Drawings of the Digital Optoelectronic Nose ${ }^{a}$
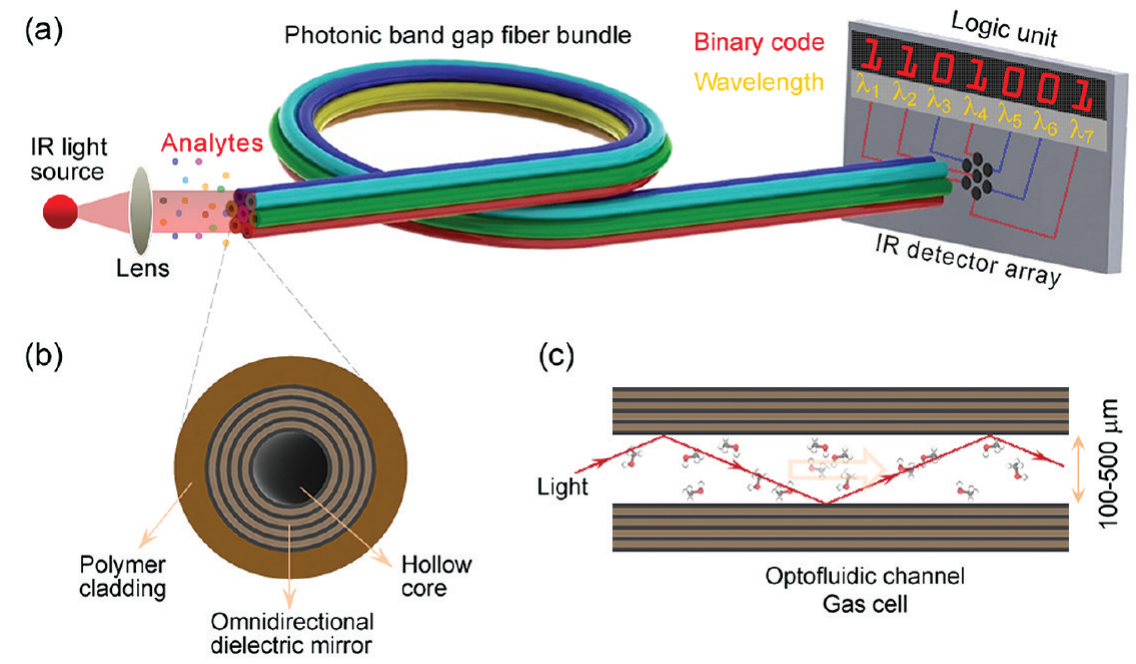

a (a) The sensor consists of an infrared (IR) source, an array of hollow core IR transmitting photonic band-gap fibers, and an IR detector array. Each fiber in the array selectively guides a narrow spectral band by filtering the incoming continuum source. The transmitting radiation interacts with odorants in the hollow core of the fiber. The quenching of the transmitted signal, due to molecular absorption, is recorded and, by comparison with a threshold value, registered as a binary code. (b) Cross section of the infrared transmitting fiber. The dielectric mirror lining the hollow core of the fiber is composed of alternating high and low index materials. The spectral filtering is tuned by the thickness of these layers during fiber production. (c) Enhanced electromagnetic interaction between the guided light and analytes present in the core of fibers (waveguides/gas cell/optofluidics channel) is achieved through the extended optical path.

parameters of the inner core structure of the fiber. Hundreds of hollow core Bragg fibers ${ }^{22}$ that span the full infrared absorption range $\left(500-4500 \mathrm{~cm}^{-1}\right)$ can be produced from a single preform by thermal drawing. ${ }^{23,24}$ By coupling to a blackbody source, such as a $\mathrm{SiC}$ rod with a peak intensity around $5 \mu \mathrm{m}$, an array of these fibers can be used to filter specific bands from the infrared continuum radiation. If the difference in the transmitted light is detected with and without the presence of volatile organic molecules in the fiber gas cell, quantitative information can be obtained about the infrared absorption of the analyte using its specific molecular absorption lines (Scheme 1).

By multiplexing wavelength selective fibers, an array of crossresponsive sensors is obtained that can be used as an artificial nose. The array can be considered cross-responsive, as is required in artificial olfaction research, ${ }^{2}$ because each fiber sensor is sensitive to a large number of molecules that have corresponding absorption peaks in the fiber transmission range, and at the same time, various analytes will result in slightly different absorption patterns depending on the exact infrared absorption characteristics and the chemical bond environment. In this paper, we show that the selectivity of this fiber array can be used to identify and distinguish a large number of volatile organic chemicals. First, the working principle of the electronic nose is demonstrated using methanol, isopropyl alcohol (IPA), and acetone analytes. By studying the in-fiber infrared absorption parameters of these analytes, we deduced simulation parameters to generalize sensor behavior as an artificial nose. The experimental results are then extrapolated with computer simulations to a data set containing 100 volatile organic chemicals. Also, the array response was used to differentiate all possible mixtures of five different chemicals. On the basis of the simulations, the correlation between the sensor array parameters and the selectivity of the fiber nose is investigated. The sensitivity, rapid response time, electric signal output, and reusability, together with high selectivity, render the fiber array suitable for electronic nose applications such as toxic gas detection, food quality control, environmental monitoring, breath analysis for disease diagnostics, health monitoring, and pharmacokinetics. $^{25}$

\section{MATERIALS AND EXPERIMENTAL METHODS}

Photonic Band-Gap Fiber Fabrication. Hollow core polymer fibers that incorporate dielectric mirrors (Scheme 1b) for infrared beam guiding are fabricated by thermal drawing using a recently developed composite preform preparation technique. ${ }^{22,23}$ The fibers in the array are produced from exactly the same type of preform using thermal drawing, as described elsewhere. ${ }^{21}$ Unlike metal-coated hollow core fibers, dielectric mirror polymers selectively guide an infrared wavelength of choice, which is determined by the size reduction factor during thermal drawing. The guided wavelength is determined by the thickness and refractive indices of the multilayers using the quarter wave stack equation $\left(\lambda=4 n_{i} d_{i}\right.$, where $\lambda$ is the wavelength of guided radiation and $n_{i}$ and $d_{i}$ are the refractive index and thickness of alternating layers). In this way, hollow core polymer fibers can be used to filter and guide continuum light from a blackbody continuum into relatively narrow wavelength bands. The fibers typically have a 100-500 $\mu \mathrm{m}$ inner core diameter and a $0.8-2.0 \mathrm{~mm}$ external diameter; they can be obtained arbitrarily long and are flexible.

In-Fiber Infrared Absorption Measurements. We used the $\mathrm{SiC}$ filament and collimator optics of an FTIR system (Bruker Tensor) and a $\mathrm{ZnSe}$ convex lens to couple the infrared beam to the hollow core of the fibers, one at a time. An $\mathrm{Hg}-$ $\mathrm{Ca}-\mathrm{Te}$ (MCT) infrared detector is used to record the total infrared energy transmitted through the fiber. The gaseous analytes were delivered into the fiber gas cell (Scheme 1c, also see Supporting Information, Figure S1) using nitrogen carrier gas from a flask saturated with the vapor pressure of the analyte gas. The flow rate of the carrier gas was controlled to be $1000 \pm$ $10 \mathrm{sscm}$ with a mass flow controller. Carrier gas was exhausted from the other end of the $\sim 30 \mathrm{~cm}$ fiber. 
(a)

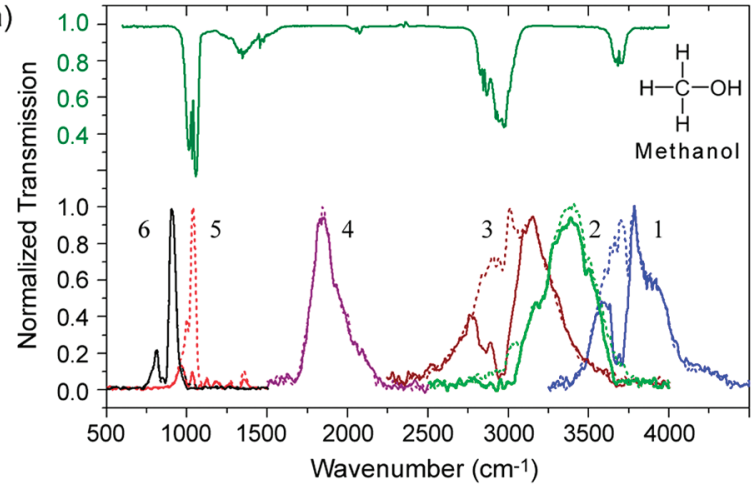

(c)

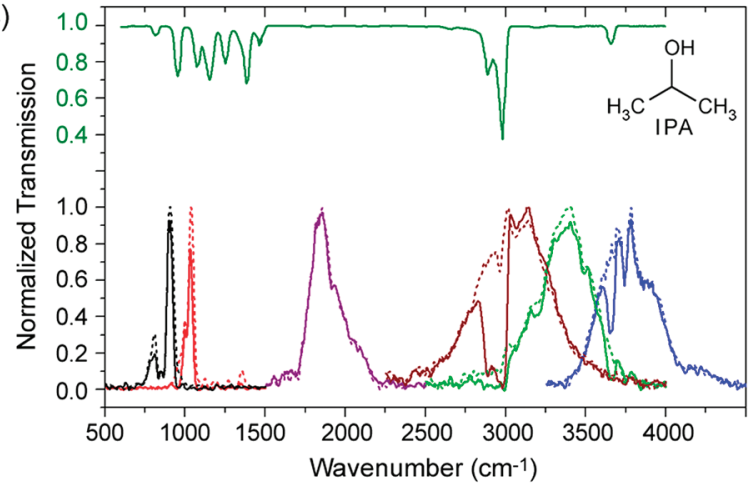

(e)

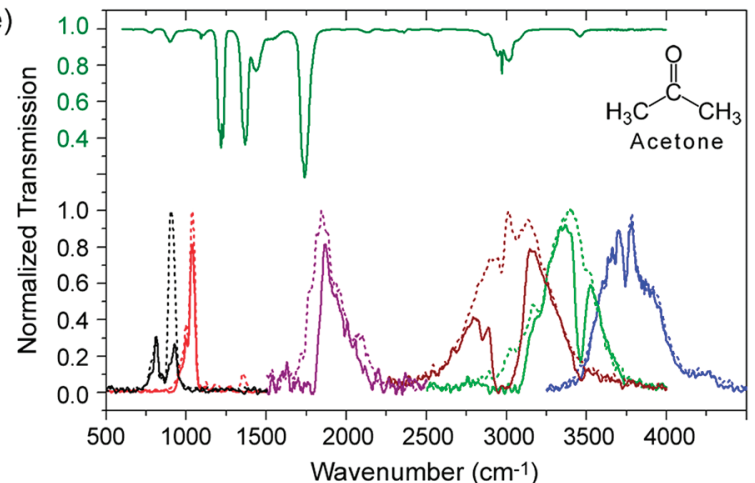

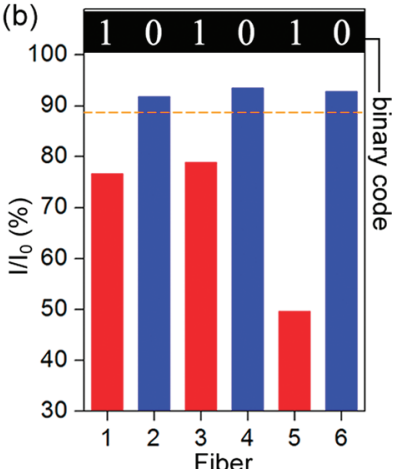
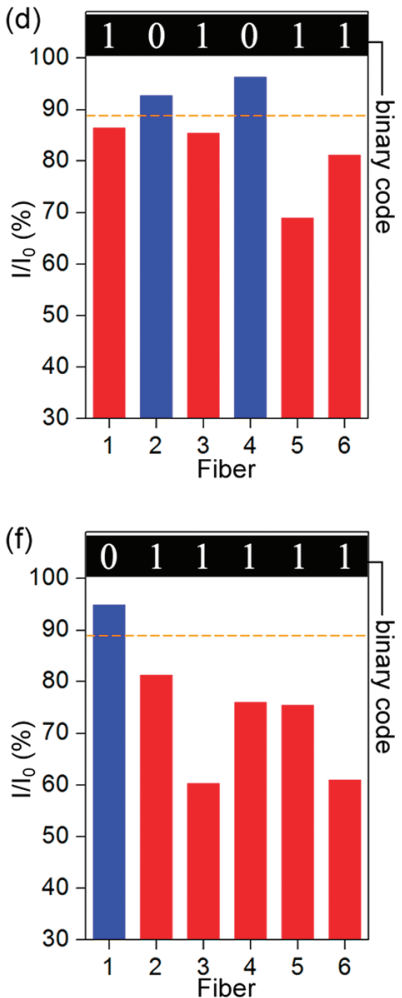

Figure 1. Response of fiber array to methanol, isopropyl alcohol (IPA), and acetone. Wavelength scalable transmission bands of a six-fiber array that have transmission bands between 4500 and $500 \mathrm{~cm}^{-1}$, and the quenching of the transmission signals due to (a) methanol, (c) IPA, and (e) acetone presence in the hollow core. Infrared absorption spectra, measured by FTIR, of methanol, IPA, and acetone are shown on the top of each figure. Total initial intensity quenches significantly for fibers 1, 3, and 5 for methanol, fibers 1, 3, 5, and 6 for IPA, and fibers 2, 3, 4, 5, and 6 for acetone. Quenching is shown as a bar chart and converted to a binary representation using a threshold value of $89 \%$. In this way, methanol is registered as "101010" (b), IPA as “101011" (d), and acetone as "011111" (f).

Fiber Array Design. A fiber sensor array of bandpass infrared transmitting fibers is used with a broadband infrared source and an integrative infrared detector. Six such fibers are selected with transmission bands at 3700, 3400, 3000, 1850, 1030 , and $910 \mathrm{~cm}^{-1}$ to cover the infrared active range of organic molecules (Figure 1). To select the spectral positions of the fibers, specific correspondence with the analytes' absorption lines is not targeted; instead, fibers are roughly placed to coincide with functional group and fingerprint region absorption. The selectivity of the system is demonstrated using methanol, IPA, and acetone at their vapor pressures, 100, 33 , and $230 \mathrm{mmHg}$, respectively. Before each gas insertion, the fiber gas cell is thoroughly flashed with nitrogen, followed by the insertion of the analyte via the carrier gas.

Simulations. We simulated quenching experiments for 100 organic volatile compounds from a commercial FTIR library
(Bruker FTIR demo library) with up to 180 computer generated fiber transmission bands. The selectivity and the parameters affecting selectivity, such the number of fibers, fiber transmission bandwidths, and threshold level settings, are investigated using the simulations. All simulations are performed using Mathematica software (version 4.0) on a desktop workstation.

\section{RESULTS AND DISCUSSION}

Array Response Data. The FTIR absorption/transmission spectra of methanol, IPA, and acetone are shown in Figure 1a, c, and e. All analytes have a $\mathrm{C}-\mathrm{H}$ absorption (around 3000 $\mathrm{cm}^{-1}$ ) and unique fingerprint region between 1500 and 500 $\mathrm{cm}^{-1}$. Besides, acetone has a carbonyl $(\mathrm{C}=\mathrm{O})$ peak near 1700 $\mathrm{cm}^{-1}$, and methanol and IPA have a hydroxyl group $(-\mathrm{OH})$ absorption near $3700 \mathrm{~cm}^{-1}$. Upon the insertion of methanol, we 
observed large quenching of the transmission signal for fibers 1 , 3 , and 5. The transmittances $(\% \mathrm{~T})$ of the fibers are 76.8, 92.0, 79.0, 93.6, 49.7, and 93.0\%, respectively. By comparing the absorption spectra with the transmittance of the fibers before and after methanol insertion, we see that the quenching in fibers is due to the absorption lines of the analyte (Figure 1a). The transmittance ratios are shown as a bar graph (Figure $1 \mathrm{~b}$, $\mathrm{d}$, and $\mathrm{f}$ ). Furthermore, by selecting a threshold value, the transmittance graph is converted to a binary representation, where the unitary binary digit represents transmittance below the threshold and a null digit above. Selecting a threshold value of $89 \%$ results in a "101010" (decimal equivalent 42 ) for methanol. For the next analytes, IPA and acetone, we observe fiber transmittances of $86.6,92.8,85.6,96.4,69$, and $81.3 \%$ and $95,81.4,60.4,76.1,75.6$, and 61.1, respectively. For IPA, fibers $1,3,5$, and 6 and, for acetone, fibers 2, 3, 4, 5, and 6 have larger quenching due to corresponding absorption peaks, as shown in Figure $1 \mathrm{c}$ and e. The same threshold value gives a binary representation "101011" (decimal equivalent 43) for IPA (Figure 1d) and "011111" (decimal equivalent 31) for acetone (Figure 1f).

The choice of a suitable threshold value and its effects are discussed below. However, it is evident that a wide threshold range can be used to differentiate these three chemicals, since the number of fibers is greatly redundant for the differentiation of just three chemicals. In fact, fibers 1,4 , and 6 can readily distinguish these three chemicals. Therefore, the extra fibers can be selected appropriately to differentiate more chemicals; for example, we recently showed that six fibers can be easily used to resolve 10 chemicals. $^{21}$ The suitable choice of a threshold value results in a unique binary fingerprint for each chemical in the measurement data set. It is essential to note that the threshold value should be wider than the standard deviation of the experimental error in the quenching values to accommodate experimental noise; that is, the binary representation for each chemical should remain unchanged due to the signal noise. We determined the statistical error in the quenching measurements by repeatedly inserting an analyte and flashing numerous times. Our results with fiber 6 with ethanol are shown in Figure S2 of the Supporting Information. After the first ethanol insertion, the transmission quenched to $74.7 \%$ of its initial value and the signal rapidly recovered to its initial value after flushing with nitrogen. In Figure S2 of the Supporting Information, eight successive measurements are shown and the statistical error in the intensity ratio was calculated to be $\pm 0.64 \%$. The permissible threshold region is defined as the band or regions of the threshold value where the binary coding of all chemicals of interest can be uniquely addressed as a binary code within the experimental error of the quenching value. From Figure 1 we deduce that any threshold value between $92 \%$ and $86.6 \%$ can be used to uniquely identify all three chemicals, within the quenching experimental error.

Sensitivity of the In-Fiber Absorption Measurements. In our previous work, ${ }^{21}$ we measured the detection limit of the photonic nose to be in the low ppm level (19 ppm for tetrahydrofuran). Although better sensitivities in the lower ppb level can be readily obtained using a laser source due to the higher optical energy density, see for example Charlton et al., ${ }^{18}$ the selectivity of the sensor is compromised when monochromatic laser sources are employed. Despite the fact that it has slightly lower sensitivity, use of a broad band light source provides high selectivity with tolerable sensitivity and enables the design of a photonic cross-responsive nose sensor.
Typical Difference Spectra. We compared the absorption peaks of the chemicals deduced from quenching measurements with typical absorption peaks seen with an FTIR instrument. The absorption peaks in the fiber cells are calculated from the difference in fiber transmission with and without the analyte (Figure 2). Chemical absorption peak positions which fall in

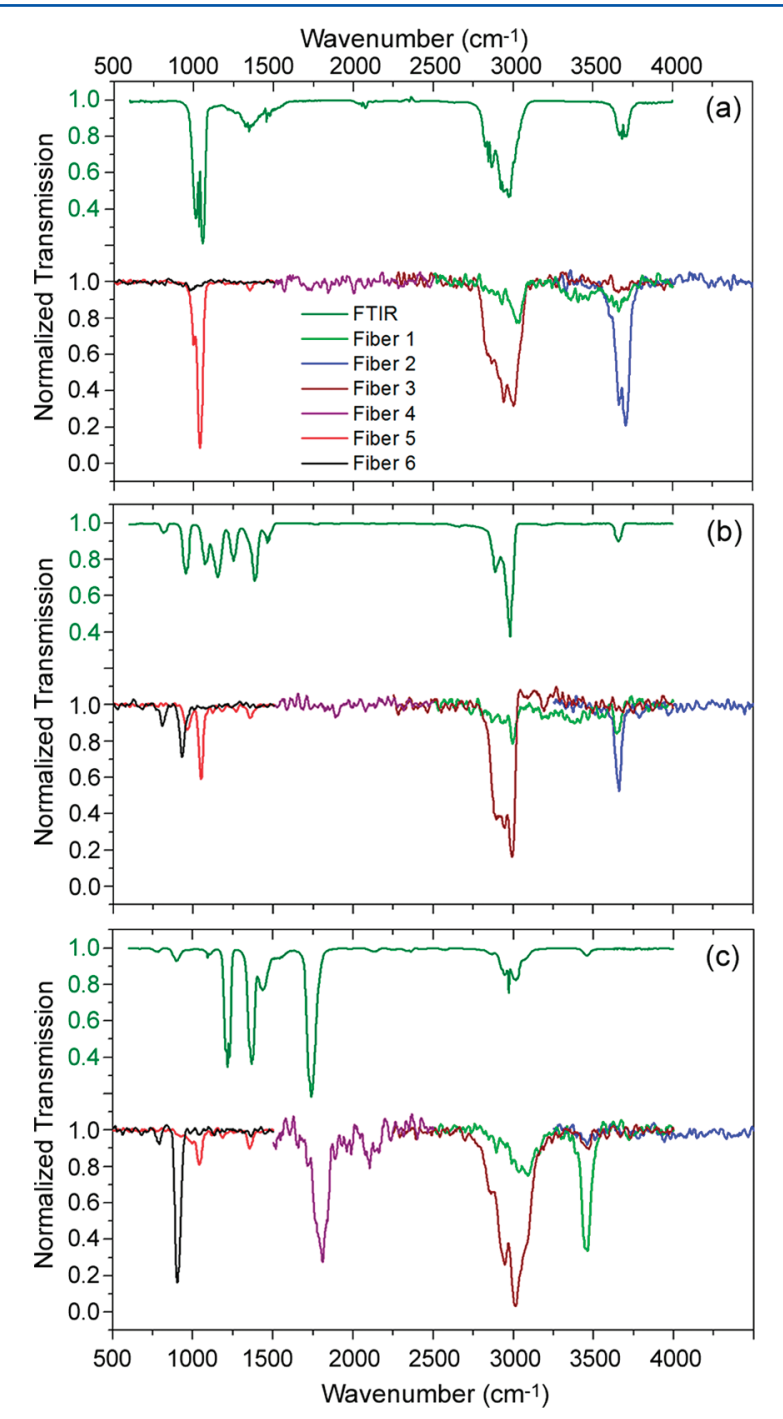

Figure 2. Difference spectra between the original and the quenched signals for each fiber in the array for (a) methanol, (b) IPA, and (c) acetone. Full FTIR absorption spectra are also shown at the top for comparison. In all cases, the difference spectra detect the presence of an absorption peak in the range of the transmission band. For example, fiber 1 successfully detects the presence of the $-\mathrm{OH}$ absorption peaks of methanol and IPA. In general, the absorption peaks detected by the fibers appear to be more pronounced compared to those of the FTIR spectra of the analyte. For example, a relatively weak absorption peak of acetone at $3450 \mathrm{~cm}^{-1}$ is shown as 15 times more intense.

the fiber transmission window clearly coincide with peaks seen using an FTIR spectrometer. Moreover, these absorption peaks appear to be more pronounced in the fiber difference spectra, probably due to the waveguiding effect and extended optical path of the infrared transmitting fiber. In Figure 2c FTIR absorption of acetone gas is compared with the difference spectra from each of the six fibers. The relatively weak peaks at 900 and $3450 \mathrm{~cm}^{-1}$ are amplified and detected by fibers 6 and 2, respectively. Pronounced absorption peaks at 2980 and 1730 
$\mathrm{cm}^{-1}$ are clearly visible. Fiber 1 has no coinciding absorption peaks and does not report any false positive signal.

To carry out infrared absorption simulations in a computer generated bandpass fiber array, a normalization factor is defined by comparing the peaks obtained from the quenching measurements with the corresponding FTIR peaks. A normalization factor (e) is calculated for each peak by taking the ratio of the corresponding peak intensities from the quenching measurement and FTIR spectra (Figure 2). We obtained normalization factors in the range $3 \times$ to $15 \times$ (Supporting Information, Table S1). For some fibers, a normalization factor could not be calculated because the transmission is completely quenched; for example, this is the case for the acetone 1700 $\mathrm{cm}^{-1}$ absorption peak using fiber 4 .

Simulations. To demonstrate the selectivity of the fiber array and the parameters affecting selectivity, we simulated quenching measurements for an expanded fiber array and analyte set. A sensor array comprising 25 photonic band-gap fibers is simulated. The fiber transmission bands are generated using a Gaussian profile similar to the real fiber transmission band shape and bandwidths. The spectral positions of the fibers are selected to roughly coincide with information bearing absorption wavelengths of the analytes (Supporting Information, Figure S3). 100 organic volatile compounds are selected from a commercial FTIR library (Bruker FTIR demo library) to test the selectivity of the array (see Supporting Information). For each fiber analyte set, the quenching is calculated from

$$
Q(i, j)=\frac{\int e_{i} A_{i}(\lambda) T_{j}(\lambda) \mathrm{d} \lambda}{\int T_{j}(\lambda) \mathrm{d} \lambda}
$$

where $e_{i}$ is the compounded normalization factor, $A_{i}(\lambda)$ is the infrared absorption of the $i^{\text {th }}$ gaseous analyte, and $T_{j}(\lambda)$ is the transmission of the $j^{\text {th }}$ fiber. We used random normalization values (e) between 3 and 15 , consistent with the upper and lower normalization values of Table S1 of the Supporting Information. In this way, a response matrix is obtained for the 25 fiber, 100 analyte set. The resulting quenching matrix is shown as a gray scale map in Figure 3a.

To convert the gray scale map to a binary representation, a threshold value is required. The threshold value is selected so that the binary representations of all analytes in the data set are distinct. Because the fiber array has redundancy in differentiating the chemicals, a band of threshold values satisfies this condition. Therefore, the threshold bandwidth is a measure of how well the fiber array differentiates the given set. In the 25 fiber, 100 chemical simulation, a threshold band between 89.2 and $92.2 \%$ is found that successfully differentiates all the chemicals in the data set. Note that the threshold band is wider than the experimental quenching error of $0.64 \%$. The response matrix is shown as a 25-digit binary code using a threshold value of $91 \%$ (Figure 3b). A full list of chemical names and their binary representations are given in Table S2 of the Supporting Information.

A distance matrix is calculated between any two chemicals from the response matrix using the Manhattan distance function

$$
L_{i, j}=\sum_{k=1}^{n}|Q(i, k)-Q(j, k)|
$$

where $n$ is the number of fibers. The distance matrix can be presented as a hierarchical clustering map which shows the (a)

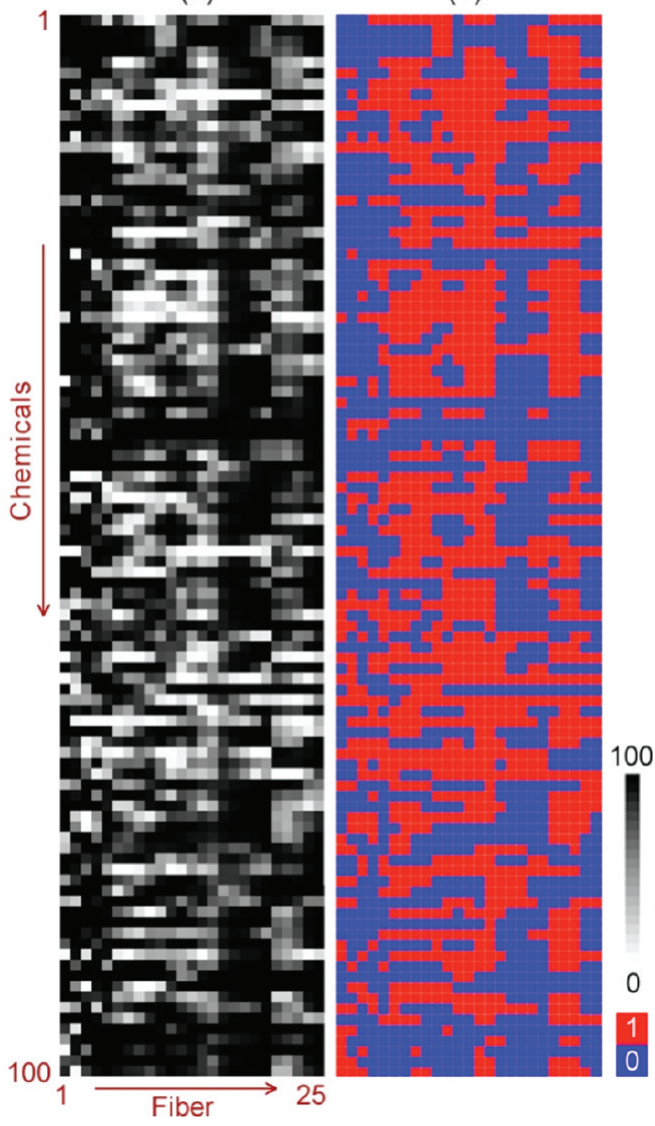

Figure 3. Response of a 25 -fiber sensor array, simulated for 100 volatile organic molecules, taken from a commercial FTIR demo library. The quenching matrix $Q(i, j)$ is shown as a gray scale representation where each chemical is represented as a row. All chemicals are successfully resolved when a threshold value of $91 \%$ is used. In this way, each chemical is represented with a unique binary number. The complete list of the successfully distinguished chemicals and their binary representation are provided in the Supporting Information (Table S2).

similarity of analytes as seen by the sensor array (Figure 4). It is interesting to compare the HCA map with the binary representations of the chemical set after ordering it according to the HCA clustering (Figure 4), where we find that chemicals are grouped according to their bond similarities. For example, chemicals \#2 (ethyl formate) and \#3 (buthyl formate) are chemically similar (only three of the 25 fibers give different signals) and located on the upper cluster, and chemicals \#30 (cyclohexanol) and \#34 (4-methylcyclohexanol) are similar and located on the bottom cluster. Also, chemicals from the first group and another from the latter (for example, \#2 and \#34) have 16 different binary signals.

Effect of Fiber Transmission Bandwidth. We investigate how fiber transmission bandwidth affects the quenching and, therefore, sensitivity, fiber array response, and selectivity. The transmission bandwidths $(\Delta \lambda)$ of photonic band-gap fibers are typically $7-8 \%$ of the transmitted wavelength $(\lambda)$ (Figure 1 ). But, if required, the transmission bandwidth can be made narrower or wider by producing fibers with slightly different Bragg layer parameters, material refractive indices, and thicknesses, or simply by bending the fiber arrays in the device. In the simulations, we tried five sets of fibers with narrower and wider bandwidths (Figure 5a). Full width half-maximum 


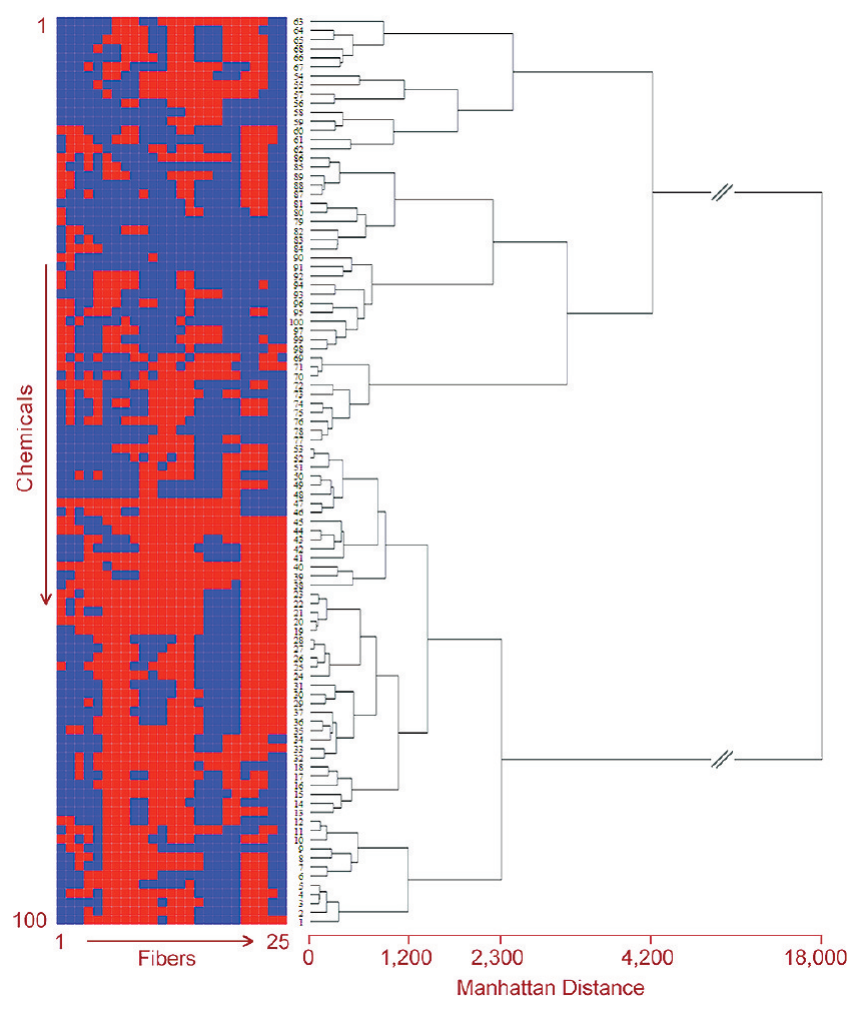

Figure 4. Hierarchical clustering analysis using the Manhattan distance matrix and a binary representation of the chemical data set. Dendrogram of the array responses to 100 common volatile organic compounds juxtaposed with the corresponding binary representation. The binary array pattern matches the dendrogram clustering, indicating the selectivity of the individual fibers in the array.

(fwhm) bandwidth values are normalized to the fwhm bandwidths of fibers used in the experiments. We observed that the width of the threshold band, for unique identification of the 100 chemicals, strongly depends on the fiber transmission bandwidths. Using computer simulations, we generated several fiber sets with narrower or wider bandwidths than those of the fibers used in experiments and repeated the simulations for each fiber set. According to Figure 5a, when the bandwidth was 1.7 or 2.5 times narrower (relative fiber bandwidths are 0.6 and 0.4 , respectively) than that of real fibers, larger threshold bands were obtained. The threshold band is observed to diminish with ever narrower bandwidths or for very wide fiber transmission bands (Figure 5a). We deduce that, for very narrow or very wide transmission bandwidths, the transmission and quenching signal-to-noise ratio decrease adversely affects sensor responsiveness. Second, and more interestingly, narrower band fibers start to lose their crossresponsiveness and could only respond to a single specific wavelength. On the other side, very wide transmission bandwidth fibers start to lose their specificity, though they appear to have a wide spectral response domain. The threshold region is a measure of the cross-responsiveness of the fiber array; as the threshold band diminishes to zero on both sides of the graph, the electronic nose becomes either too specific or insensitive.

The fiber array has practically unavoidable redundancy in the number of fibers used to distinguish the chemical data set. In theory, $n$ fibers could register $2^{n}$ chemicals. However, if this is targeted, the electronic nose would become a specifically designed chemical sensor for each chemical in the set. In reality,
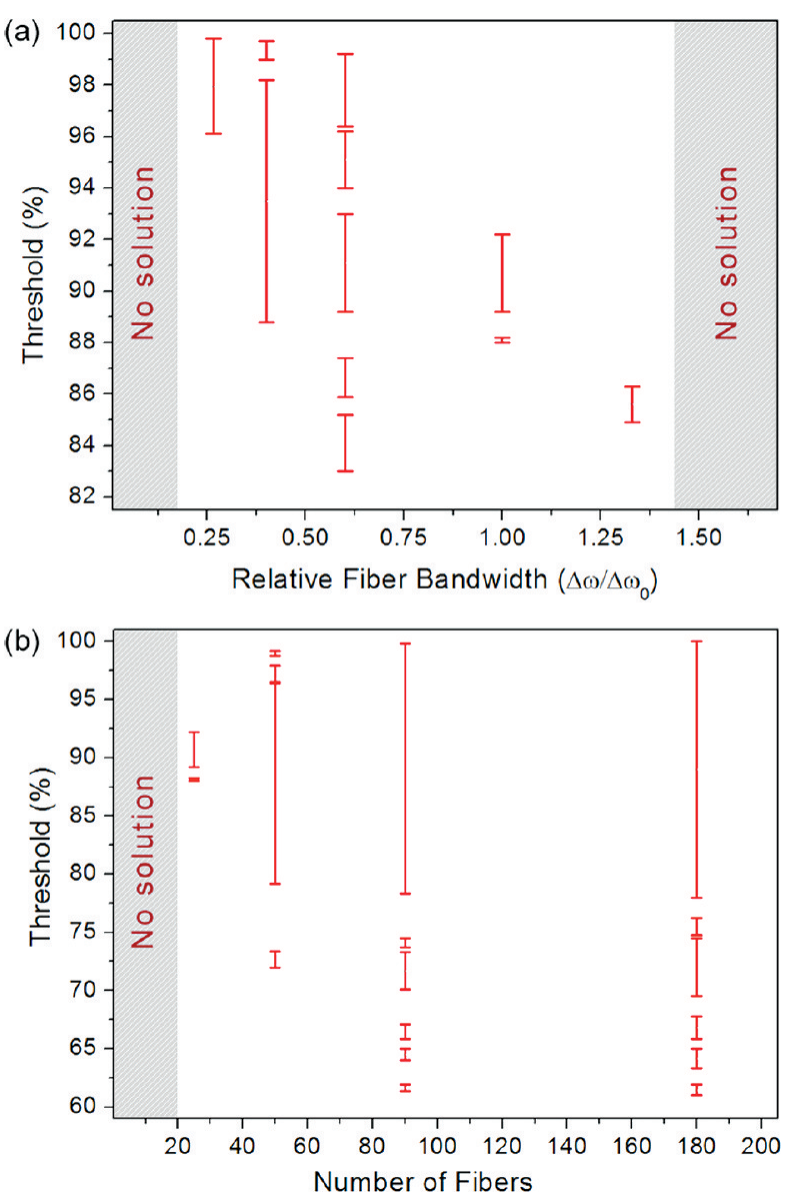

Figure 5. (a) Threshold bands are shown for five different fiber sets that have same spectral positions, but narrower or wider transmission bandwidths (fwhm). The fibers used in the experiments are taken as unity in the relative bandwidth scale. The widest continuous threshold band is observed for the $2.5 \times$ relative bandwidth, and the threshold region vanishes for too narrow and too wide fibers, as indicated. All threshold bands are calculated for the same 100 chemicals. (b) Dependence of the selectivity threshold bands on the number of fibers. When 25 fibers are used, the threshold band is wide enough (4\%) to selectively discriminate all 100 chemicals. The threshold region widens for 50 and 90 fibers, but a further increase in fiber numbers introduces redundancy and does not significantly affect it.

electronic noses are designed to feature broad selectivity and high sensitivity.

Effect of the Number of Fibers on the Threshold Band. It is shown that 25 fibers were adequate to distinguish the selected data set of 100 chemicals. The threshold band obtained extends $3 \%$ from 89.2 to $92.2 \%$ (Figure 5 b). We further investigated how the threshold band changes with the addition of extra fibers. If a fiber set already distinguishes each analyte distinctly, then further addition of fibers will increase redundancy and widen the possible threshold band. We show the effective threshold bands for 25, 50, 90, and 180 fibers. The spectral positions of these fibers are selected so that they are evenly placed in the absorption regions of the spectra. A full list of the spectral positions of the fibers is given in the Supporting Information.

The threshold bandwidth almost quadruples if 50 fibers are used, but band-gaps appear between allowable threshold regions. For 90 fibers, the threshold region covers nearly $40 \%$ 
of all possible threshold values and does not change much with the addition of another 90 fibers.

Recognizing Mixtures. The fiber array response in the presence of mixtures is investigated by using 25 normal bandwidth fibers. Five different analytes, 1-dimethyl formamide (DMF), 2-ethanol, 3-methanol, 4-acetone, and 5-toluene, and all their 26 possible mixtures were simulated to obtain the response matrix. Each unary, binary, ternary, quaternary, and quinary mixture was treated as a separate chemical; a full list is given in the Table S3 of the Supporting Information. We assume the chemicals do not interfere with each other's absorption characteristics.

The simulations show that it is possible to differentiate chemicals and their mixtures within a threshold region of 77$85 \%$. All 31 elements are uniquely identified with a threshold value of $80 \%$ using the binary coding scheme (Figure 6). In this

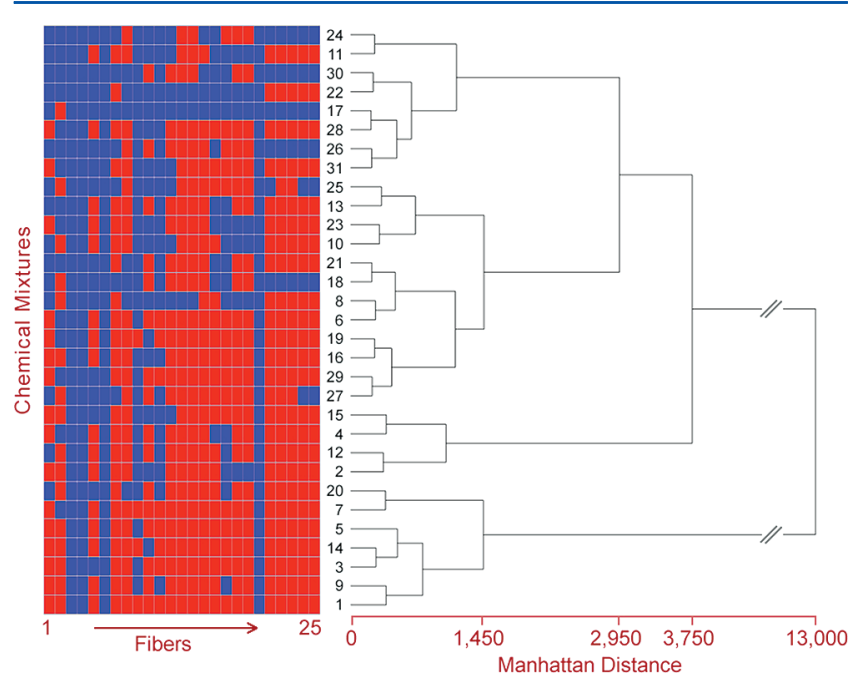

Figure 6. (a) Binary representation of all possible combinations of five chemicals from the same chemical set Table S3 of the Supporting Information. Chemical mixes are differentiated using a threshold value of $80 \%$. According to the simulations, 9 of the 25 fibers are redundant; 16 fibers are enough to successfully distinguish the mixings. (b) Hierarchical clustering representation using the Manhattan distance matrix.

simulation 25 fibers are used, however. The fiber binary coding scheme indicates that some fibers give exactly the same response. For example, the fiber responses of 7, 4, and 25 are the same for all 31 elements. Thus, removing two of these fibers will not affect selectivity. Also, four of the fibers $(3,4,6,20)$ are always off for all chemicals at this threshold. By removing these redundancies, we observed that 16 fibers successfully resolve the selected five chemicals and all their mixtures. The hierarchical clustering representation is drawn using the Manhattan distance function (Figure 6).

\section{CONCLUSION}

Six typical Bragg fibers are fabricated, and controlled in-fiber infrared absorption detection of volatile analytes is carried out. On the basis of the experiments, a computer simulation is designed to extend the number of fibers and chemicals to quantitatively investigate the selectivity of the scheme. Using the simulations, it was observed that a large set of 100 chemicals can be identified from the binary sampling of their characteristic infrared absorption peaks using the fiber based electronic nose scheme. The high selectivity of the nose scheme is highlighted. Sampling the absorption spectrum using finite bandwidth fibers enables binary representation of the resulting response matrix. It was observed using simulations that the transmission bandwidth is critical in order to devise crossresponsive sensors. If the bandwidth is too narrow, fibers become too specific; if it is too broad, they lose specificity. In this way, we effectively quantified the cross-responsiveness of the digital nose in terms of its selectivity.

Theoretically, using an $n$-fiber array electronic nose, $2^{n}$ analytes could be registered, provided there is no redundancy in the fiber array. Redundancy in the system is defined as the condition in which two or more fiber's binary responses are the same for all chemicals in question. Practically, redundancy is inevitable with finite bandwidth fibers; moreover, we demonstrated that this redundancy is required for electronic nose applications. The redundancy in the fibers is a key aspect of the cross-responsive nature of the sensors. Simulations also indicate that the usable threshold band narrows when intractable chemicals, those very similar with respect to their absorption spectra, as seen by the sensor array, are included. This can be avoided by addressing extra fibers for these intractable chemicals or, alternatively, by engineering the photonic band gap.

The selectivity of the fiber sensors and their cross-responsive nature was shown to be appropriate for the detection of volatile organics and, therefore, as an electronic nose. Additional unique features such as the electrical signal output, real time response, reusability, low cost, and low power consumption make a photonic fiber nose a potentially interesting contender as an artificial nose device.

\section{ASSOCIATED CONTENT}

\section{Supporting Information}

A scheme of the experimental set up and a figure showing the repeatability of the quenching response of the nose. Detailed simulation results of the fiber array based optoelectronic nose system and the complete list of the successfully distinguished chemicals and their binary representations. This material is available free of charge via the Internet at http://pubs.acs.org.

\section{AUTHOR INFORMATION}

\section{Corresponding Author}

*E-mail: bayindir@nano.org.tr. Telephone: (+90) 312290 3500. Fax: (+90) 3122664365.

\section{ACKNOWLEDGMENTS}

This work was partially supported by the Ministry of Development and TUBITAK under Project No. 110M412. M.B. acknowledges support from the Turkish Academy of Sciences Distinguished Young Scientist Award (TUBA GEBIP).

\section{REFERENCES}

(1) Zarzo, M. Biol. Rev. Cambridge Philos. Soc. 2007, 82, 455-475.

(2) Pearce, T. C.; Schiffman, S. S.; Nagle, H. T.; Gardner, J. W. Handbook of Machine Olfaction; Wiley-VCH: Weinheim, 2003.

(3) Persuad, K.; Dodd, G. Nature 1982, 299, 352.

(4) McAlpine, M. C.; Ahmad, H.; Wang, D.; Heath, J. R. Nat. Mater. 2007, 6, 379-384.

(5) Peng, G.; Tisch, U.; Adams, O.; Hakim, M.; Shehada, N.; Broza, Y. Y.; Billan, S.; Abdah-Bortnyak, R.; Kuten, A.; Haick, H. Nat. Nanotechnol. 2009, 4, 669-673. 
(6) Lonergan, M. C.; Severin, E. J.; Doleman, B. J.; Beaber, S. A.; Grubbs, R. H.; Lewis, N. S. Chem. Mater. 1996, 8, 2298-2312.

(7) Grate, J. W. Chem. Rev. 2000, 100, 2627-2648.

(8) Lange, D.; Hagleitner, C.; Hierlemann, A.; Brand, O.; Baltes, H. Anal. Chem. 2002, 74, 3084-3095.

(9) Dickinson, T. A.; White, J.; Kauer, J. S.; Walt, D. R. Nature 1996, 382, 697-700.

(10) Aernecke, M. J.; Walt, D. R. Sens. Actuators, B: Chem. 2009, 142, 464-469.

(11) Aernecke, M. J.; Walt, D. R. Anal. Chem. 2009, 81, 5762-5769.

(12) Rakow, N. A.; Suslick, K. S. Nature 2000, 406, 710-713.

(13) Lim, S. H.; Feng, L.; Kemling, J. W.; Musto, C. J.; Suslick, K. S. Nat. Chem. 2009, 1, 562-567.

(14) Janzen, M. C.; Ponder, J. B.; Bailey, D. P.; Ingison, C. K.; Suslick, K. S. Anal. Chem. 2006, 78, 3591-3600.

(15) Bonifacio, L. D.; Puzzo, D. P.; Breslav, S.; Willey, B. M.; McGeer, A.; Ozin, G. A. Adv. Mater. 2010, 22, 1351-1354.

(16) Monat, C.; Domachuk, P.; Eggleton, B. J. Nat. Photonics 2007, 1, $106-114$.

(17) Skoog, D. A.; West, D. M.; Holler, F. J. Analytical chemistry: an introduction, 7th ed.; Saunders College Pub.: Philadelphia, 2000.

(18) Charlton, C.; Temelkuran, B.; Dellemann, G.; Mizaikoff, B. Appl. Phys. Lett. 2005, 86, 194102-194104.

(19) Adato, R.; Yanik, A. A.; Amsden, J. J.; Kaplan, D. L.; Omenetto, F. G.; Hong, M. K.; Erramilli, S.; Altug, H. Proc. Natl. Acad. Sci. U.S.A. 2009, 106, 19227-19232.

(20) Kim, S.-S.; Young, C.; Mizaikoff, B. Anal. Bioanal. Chem. 2008, 390, 231-237.

(21) Yildirim, A.; Vural, M.; Yaman, M.; Bayindir, M. Adv. Mater. 2011, 23, 1263-1267.

(22) Yeh, P.; Yariv, A.; Marom, E. J. Opt. Soc. Am. 1978, 68, 11961201.

(23) Temelkuran, B.; Hart, S. D.; Benoit, G.; Joannopoulos, J. D.; Fink, Y. Nature 2002, 420, 650-653.

(24) Abouraddy, A. F.; Bayindir, M.; Benoit, G.; Hart, S. D.; Kuriki, K.; Orf, N.; Shapira, O.; Sorin, F.; Temelkuran, B.; Fink, Y. Nat. Mater. 2007, 6, 336-347.

(25) Seong-Soo, K.; Young, C.; Vidakovic, B.; Gabram-Mendola, S.; Bayer, C. W.; Mizaikoff, B. IEEE Sens. J. 2010, 10, 145-158. 